\title{
Object Transport by Modular Robots that Self-assemble
}

\author{
Roderich Groß, Elio Tuci, Marco Dorigo \\ IRIDIA \\ Université Libre de Bruxelles \\ 1050 Brussels, Belgium \\ Email: $\{$ rgross, etuci, mdorigo\}aulb.ac.be
}

\author{
Michael Bonani, Francesco Mondada \\ Autonomous System Lab (ASL) \\ École Polytechnique Fédérale de Lausanne \\ 1015 Lausanne, Switzerland \\ Email: $\{$ firstname.lastname\}@epfl.ch
}

\begin{abstract}
We present a first attempt to accomplish a simple object manipulation task using the self-reconfigurable robotic system swarm-bot. The number of modular entities involved, their global shape or size and their internal structure are not pre-determined, but result from a self-organized process in which the modules autonomously grasp each other and/or an object. The modules are autonomous in perception, control, action, and power. We present quantitative results, obtained with six physical modules, that confirm the utility of self-assembling robots in a concrete task.
\end{abstract}

\section{INTRODUCTION}

Modular robotics is still progressing very quickly and holds the promise of more flexible and robust robots [1], [2]. A modular robot is composed of autonomous modules that can be connected in many different ways. Typically, the number of module types is far less than the number of modules. In some systems, the structural reconfiguration of robots is carried out manually [3], [4]. Self-reconfigurable robots, in contrast, feature the ability to autonomously change the structure in which modules are connected [5]-[8]. Based on the nature of reconfiguration, most systems can be classified as chain, lattice, or mobile reconfigurable systems [1]. While in the first two types of systems, each module usually remains connected to the modular robot at one or more points, mobile modular systems self-reconfigure by having modules detach themselves from the modular robot and move independently to another location to reconnect.

Self-reconfigurable robots have proven capable of selfrepair [9], [10], self-assembly [11]-[14], and locomotion on a plane [15], [16] or all-terrain [7], [17]-[19].

Little attention has been paid to self-reconfigurable robots that interact with their environment [20]-[22]. Some works addressed object manipulation by modular robots that were stationary and had explicit knowledge about the position of the object to manipulate [15], [23]. Kotay and Rus [24] designed a modular robot called Inchworm consisting of two foot modules and two leg modules, that were manually assembled. Although the modules could not self-reconfigure, it was shown that the robot could grasp an object with one foot module while using the other foot module to maintain a stable attachment to the surface. Thereby, the robot could manipulate the grasped object by exerting forces.

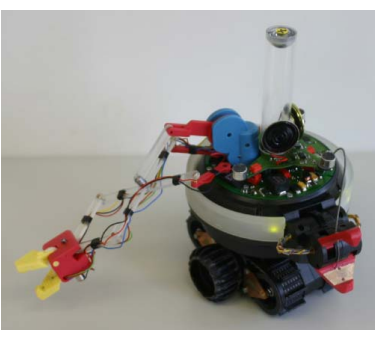

(a)

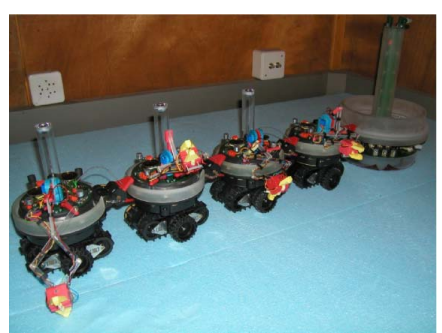

(b)
Fig. 1. The swarm-bot concept: (a) a mobile module called $s$-bot, fully autonomous in perception, control, action, and power, capable of approaching and assembling with its teammates, (b) a modular robot called swarm-bot, composed of four s-bots, manipulating an object.

In this paper, we address a simple manipulation task by a non-stationary, self-reconfigurable robot (see Fig. 1). The task consists in the transport of a heavy object to a target zone. The task is such that it cannot be solved by a 1-module robot alone. The control of the modules is decentralized and homogeneous. The modules are not provided with any explicit knowledge about the structure of the environment, such as the position of modules, the object, or the target zone.

The paper is organized as follows. Section II introduces the experimental system. In Section III we study the manipulation of an object by modular robots that are manually arranged and connected to the object by the experimenter. We analyze the impact of the frictional forces between the modules and the ground. Moreover, we analyze the impact of the spatial arrangements of the modules. In Section IV, we study situations in which the modules are initially randomly scattered in the environment. We aim at controlling the modules so that they autonomously form modular robots which in turn manipulate the environment.

\section{System Design}

We are using a mobile self-reconfigurable robot called swarm-bot [25], [26]. The modules comprising a swarmbot, called s-bots, are fully autonomous and mobile. S-bots can self-assemble, that is, they can autonomously connect to each other to form a modular robot. Self-assembly has been extensively studied on different types of terrain and with up to 16 fully autonomous, physical s-bots [14]. 
In the following the s-bot's hardware and control are detailed.

\section{A. Hardware Design}

Fig. 1a shows the physical implementation of the s-bot. It has a height of $19 \mathrm{~cm}$ (in total) and weighs approximately $700 \mathrm{~g}$.

The s-bot has nine degrees of freedom (DOF), all of which are rotational, including two DOF for the traction system, one DOF to rotate the s-bot's upper part (called the turret) with respect to the lower part (called the chassis), one DOF for the grasping mechanism of the rigid gripper (in what we define to be the s-bot's front), and one DOF for elevating the arm to which the rigid gripper is attached (e.g., to lift another s-bot). The s-bot's traction system consists of a combination of tracks and two external wheels, called treels ${ }^{\odot}$. When connected in a group, the chassis of an s-bot can be oriented in any (horizontal) direction. This allows for a coordinated motion of the modules in the group. The s-bot is equipped with a surrounding ring matching the shape of the gripper (see Fig. 1). This makes it possible for the s-bot to receive connections on more than two thirds of its perimeter. For the purpose of robotrobot communication, the s-bot has been equipped with eight RGB LEDs distributed around the turret.

The s-bot is equipped with a variety of sensors, including 15 proximity sensors distributed around the turret that sense for other objects in a $15 \mathrm{~cm}$ range, a force sensor between the turret and the chassis, and an omni-directional VGA camera to perceive the RGB LEDs of other s-bots and of the transport object. The camera can also be used to detect the direction of the light source. Two optical light barriers are integrated in the gripper to test whether an object to grasp is present or not. Furthermore, proprioceptive sensors provide internal motor information such as the torque acting on each side of the traction system.

The s-bot runs a Linux operating system on an X-Scale processor at $400 \mathrm{MHz}$. A $10 \mathrm{Wh}$ Lithium-Ion battery provides more than two hours of autonomy. For a more comprehensive description of the s-bot's hardware see [26].

\section{B. Control Design}

We aim at controlling a group of s-bots in fully autonomous manner to transport a heavy object towards a target location, indicated by a light source.

The control system described in this section has been previously designed in a relatively simple simulation environment [27], and subsequently transferred to the real sbot. The control is decentralized (i.e., fully distributed) and homogeneous (i.e., all group members have identical control). It comprises two sub-modules: (i) the "assembly" module, a simple neural network based solution which is in charge of controlling the s-bot until it has connected to the object either directly, or by becoming part of a modular robot that is connected to the object; and (ii) the "transport" module, which allows the s-bot to move the object towards the target zone once a connection is established. The transport module

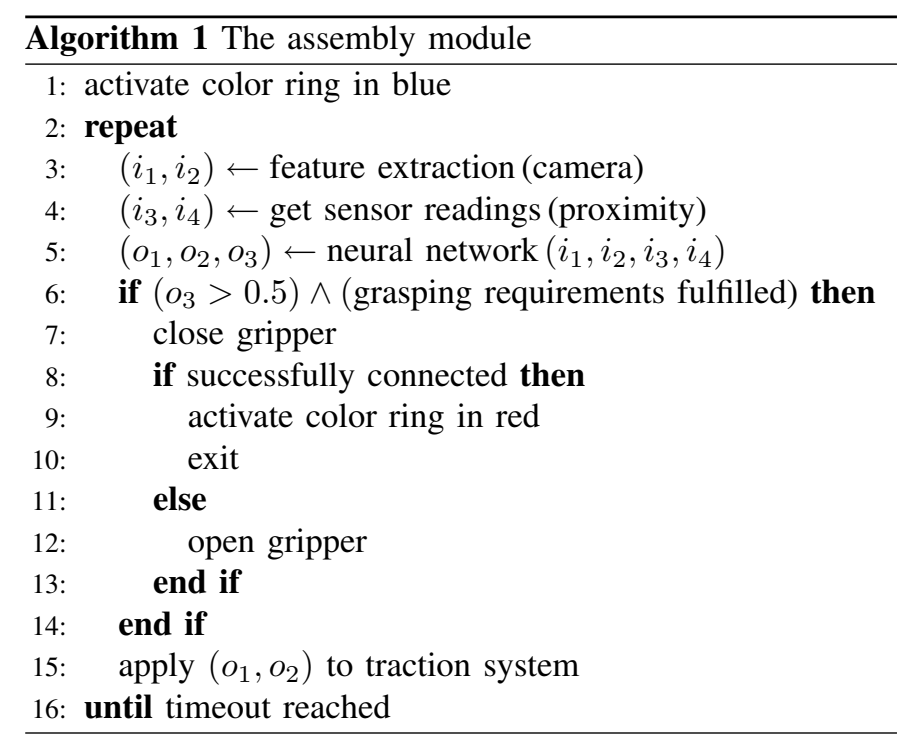

consists of two sub-modules: a simple, but effective, handcoded algorithm for controlling s-bots capable of perceiving the angular position of the target, and a recurrent neural network controller that makes use of torque and force sensors for controlling s-bots incapable of perceiving the target. The weights of both neural networks, that is, the one for selfassembly and the one for transport, were shaped in a single artificial evolution in the context of a cooperative transport task in simulation [27].

In the two experimental studies described in this paper, only the control module for self-assembly and the simple, handcoded transport module are employed. In fact, we assume that every s-bot perceives the target at each control cycle. S-bots that temporarily can not perceive the target during transport are programmed to suspend motion. ${ }^{1}$

1) Assembly Module: We aim at controlling a group of sbots in a fully autonomous manner in such a way that they locate, approach, and connect directly with an object that acts as a seed or with other $s$-bots already connected to the seed.

The process of self-assembling is governed by the attraction and repulsion among s-bots, and between s-bots and the seed. The seed object is equipped with a color ring that is permanently activated in red. Initially, all s-bots set the ring color to blue. The controller lets the s-bots avoid blue objects, and approach/connect with red objects. Thus, the process is triggered by the presence of the seed. Once an s-bot has established a connection, the color of its ring is set to red. Therefore, it becomes itself an object with which to establish a connection. The basic principle of signaling the state (of being connected or unconnected) allows the emergence of (global) connection patterns of dimensions beyond the s-bot's (local) sensing range.

Algorithm 1 details the control module for self-assembly. Its main component is a feed-forward two-layers artificial neural

\footnotetext{
${ }^{1}$ The more elaborate solution (i.e., the neural network sub-module [27]) has also been transferred to the real s-bot, and extensively tested in a dedicated experiment [28].
} 


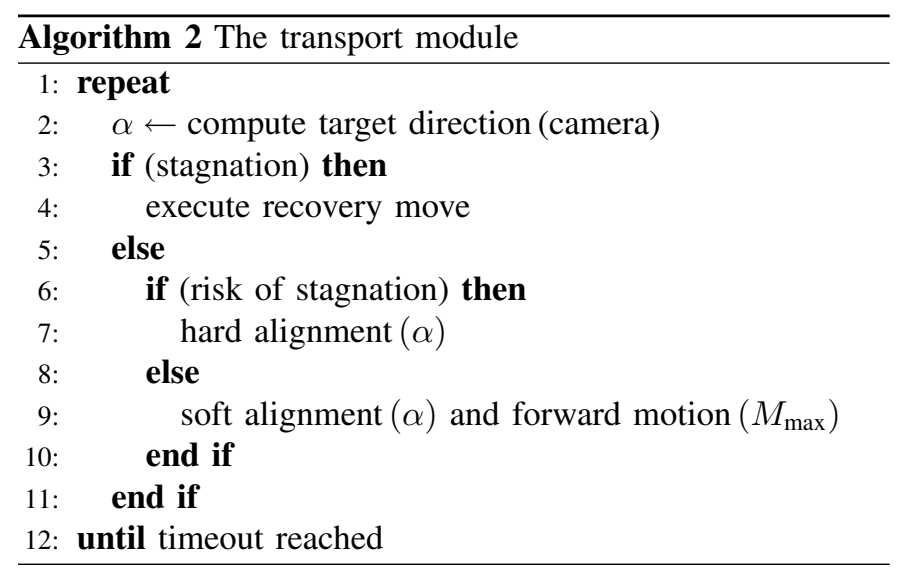

network (line 5) that maps sensory inputs to motor commands. At each control cycle, the network takes input from the s-bots vision system (line 3), and from the front-left-side and frontright-side s-bot's proximity sensors (line 4). The network's output is used to control the speed of the left and the right side of the traction system (see line 15) and the connection mechanism (see lines 6 to 14). For a more comprehensive description of the assembly module see [14], [29].

2) Transport Module: The transport module allows a connected s-bot to align its chassis towards the light source indicating the target, and to apply pushing/pulling forces in order to move the object towards the target. It is detailed in Algorithm 2.

During the transport, the s-bot monitors the magnitude of the torque acting on its traction system and on the turret. If the torque reading values exceed a certain threshold, there is stagnation. In this case, a short recovery move is performed to prevent the hardware from being damaged.

The transport module uses the camera vision system to detect the direction of the light source with respect to the sbot's heading. By adjusting the orientation of the chassis with respect to the s-bot's heading (i.e., the orientation of the turret) the controller sets the direction of motion $\alpha$. The realignment of the chassis is supported by the motion of the traction system. We implemented two different types of realignment referred to as "hard" and "soft" alignment. The hard alignment makes the s-bot turn on the spot. The soft alignment makes the s-bot turn while moving forward with maximum speed $\left(M_{\max }\right)$. The hard alignment is executed if there is risk of stagnation. This is the case, for instance, if the angular mismatch between the current and the desired orientation of the chassis exceeds a certain treshold.

\section{TRANSPORT BY MODULAR RoBOTS}

In this section we address a simple object manipulation task by modular robots consisting of s-bots that are manually connected to the object by the experimenter. The more complex situation in which the modular robots self-assemble before manipulating the object is considered in the subsequent section.

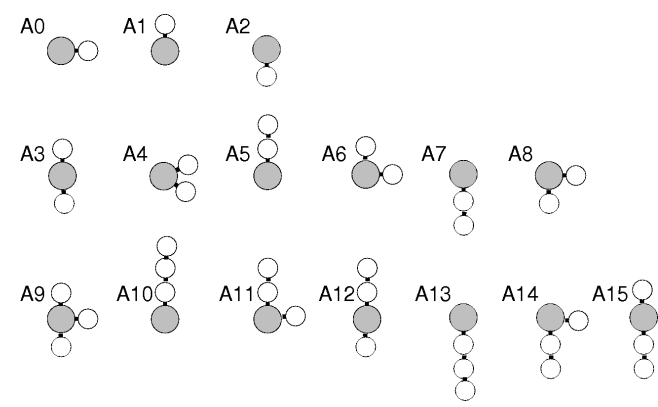

Fig. 2. Experimental setup. An object has to be transported by pulling/pushing it, towards a target (on the right side, not shown). The physical s-bots are manually attached to the object in one of the spatial arrangements illustrated in the figure. In each arrangement, every s-bot has visual contact with the target.

TABLE I

FRICTION COEFFICIENTS FOR THE GROUNDS $G_{0}$ AND $G_{1}$.

\begin{tabular}{|l|c|c|c|}
\hline & object & s-bot (lateral) & s-bot (longitudinal) \\
\hline ground $G_{0}$ & 0.46 & 0.57 & 0.58 \\
\hline ground $G_{1}$ & 0.41 & 1.30 & 1.80 \\
\hline
\end{tabular}

The task is to pull or push the object towards a light source. We analyze the impact of frictional forces between the modular robots and the ground. Moreover, we examine the performance exhibited by the modules when being arranged in different connection patterns.

\section{A. Experimental Setup}

We examine the transport of an object by a homogeneous group of physical s-bots (see Fig. 1b). The object has a weight of $813 \mathrm{~g}$. It has to be transported by pulling/pushing it towards a target (i.e., a light source). Object and target are placed at the opposite sides of an arena. The initial distance between the object and the target is $250 \mathrm{~cm}$.

In this study, groups of 1 to 3 physical s-bots are used. The s-bots are manually connected to the object from the beginning. An s-bot can connect either directly to the object or by becoming part of a modular robot, also called swarmbot, that is connected to the object. We studied the 16 distinct spatial arrangements $\left(A_{0}, A_{1}, \ldots, A_{15}\right)$ illustrated in Fig. 2. All arrangements ensure that at the beginning the target is visible for each s-bot. ${ }^{2}$ The s-bots do not have any knowledge about their spatial arrangement.

We examine the performance of the system on two different grounds $\left(G_{0}\right.$ and $\left.G_{1}\right)$. Both grounds are flat, the friction coefficients are listed in Table I. We consider the friction coefficients for ground $G_{0}$ as moderate. For ground $G_{1}$, however, horizontal forces manually applied to a non-moving s-bot cause the s-bot either to topple down or to displace by a sequence of irregular movements. Therefore, we consider

\footnotetext{
${ }^{2}$ At the time we carried out this particular experiment, the s-bot camera device driver was not yet available. Instead, the proximity sensors have been used to detect the target direction. Contrary to the omni-directional camera, the proximity sensors cannot perceive the target location if an s-bot is located in between. For the experiment described in section IV, the camera was used.
} 


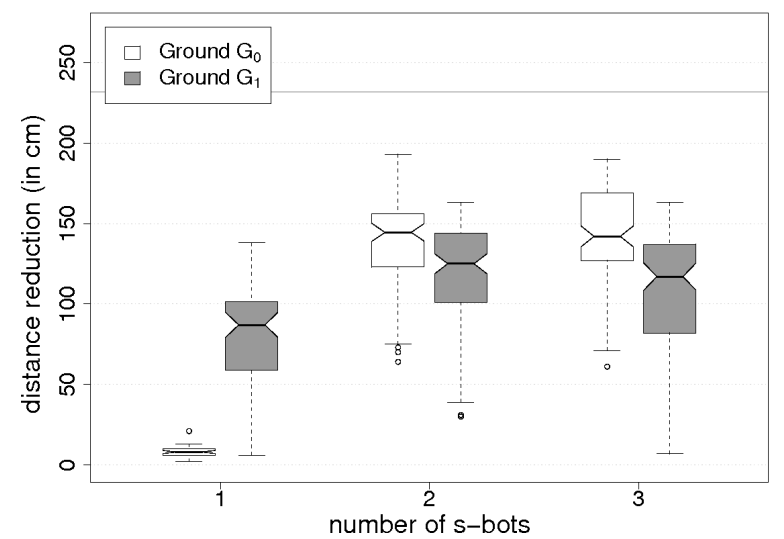

Fig. 3. Box-and-whisker plot [30] showing the observed distances (in $\mathrm{cm}$ ) by which the object approached the target during the test period of $15 \mathrm{~s}$. Observations are grouped according to the number of s-bots and the type of ground used. Observations per box (from the left to the right): 42, 75, 90, 120,105 , and 105. The horizontal line on top indicates an upper bound for the transport performance assuming a weightless object (for details see text). If the notches of two plots do not overlap, this is strong evidence that the two medians differ [30].

ground $G_{1}$ as a very difficult test-bed for studying locomotion and transport with groups of connected s-bots.

\section{B. Experimental Results}

To assess the performance of the physical system on the grounds $G_{0}$ and $G_{1}$, in total more than 500 trials were performed. Each trial lasts $15 \mathrm{~s}$. Our performance metric is the difference between the distance from the object to the target at the start of the trial and the distance from the object to the target at the end of the trial.

The distance an s-bot can cover on ground $G_{0}$ or $G_{1}$ during the time period of $15 \mathrm{~s}$ is about $232 \mathrm{~cm}$. In this case, the maximum speed of the traction system is chosen as for the transport controller $\left(M_{\max }\right)$. On ground $G_{0}$ an s-bot can move the object within $15 \mathrm{~s}$ for about $8 \mathrm{~cm}$ by pulling it backwards with maximum speed. However, a chain of two s-bots can pull the object for about $210 \mathrm{~cm}$. Since a group cannot transport a load faster than the maximum speed of each group member, a chain of two s-bots is sufficient for reaching near optimal performance (i.e., $91 \%$ of the theoretical upper bound).

Fig. 3 plots the distance (in $\mathrm{cm}$ ) by which the object approached the target. The white boxes refer to the transport performance of groups of 1 to 3 s-bots on ground $G_{0}$. In all trials, one s-bot alone was nearly incapable of moving the object. On the contrary, two and three s-bots have transported the object during each of the 90 trials for more than $60 \mathrm{~cm}$. The whiskers of the plot cover observations in the intervals $[2,13],[75,193]$ and $[71,190]$, respectively.

The gray boxes in Fig. 3 refer to the transport performance of groups of 1 to 3 s-bots on ground $G_{1}$. The whiskers cover observations in the intervals [6, 138], [39, 163] and [7, 163].

Due to the better grip the traction system has on ground $G_{1}$, a single s-bot itself is already capable of transporting the object. Nevertheless, for the group sizes 2 and 3 the system performs significantly better on ground $G_{0}$. Note that the

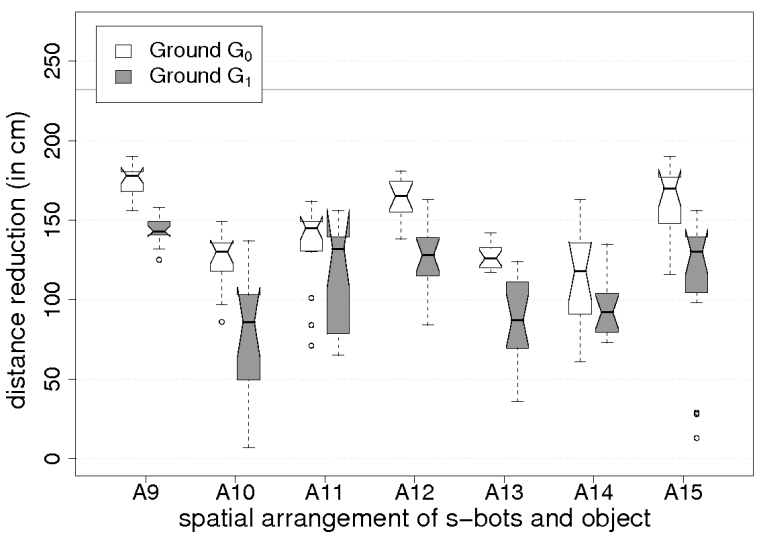

Fig. 4. Box-and-whisker plot [30] showing the observed distances (in $\mathrm{cm}$ ) by which the object approached the target during the test period of $15 \mathrm{~s}$. Observations of three s-bot experiments grouped according to the spatial arrangement used (see Fig. 2). Each box comprises 15 observations. If the notches of two plots do not overlap, this is strong evidence that the two medians differ [30].

magnitude of the force necessary to move the object on ground $G_{0}$ is slightly bigger than on ground $G_{1}$ (see Table I).

As discussed previously, the task can be solved near optimally by two s-bots. There seems to be no gain in performance by adding the third s-bot. On the other hand, the third s-bot does not disrupt the performance either.

In the following we examine the results for groups of three s-bots in more detail. The box-and-whisker plot shown in Fig. 4 groups observations belonging to the same spatial arrangement. The white boxes refer to trials performed on ground $G_{0}$, while the gray ones refer to trials performed on ground $G_{1}$. For all different spatial arrangements, the median performance on ground $G_{0}$ is superior to the median performance on ground $G_{1}$. Although the performance of a single s-bot is superior on ground $G_{1}$, which provides the s-bot's traction system with a better grip, a group of s-bots performs better on ground $G_{0}$. On ground $G_{0}$ the traction system of the s-bot may slide more easily than on ground $G_{1}$. Therefore, the performance is less affected by individual misalignments of the traction system.

By comparing the patterns of the white and gray boxes, it can be recognized that the spatial arrangement of the s-bots affects the performance. Overall, it seems that the arrangements $A_{9}, A_{12}$, and $A_{15}$, which are those in which at least one s-bot is located on both sides of the object (with respect to the target) result in a better performance than the others. This is plausible, since in these arrangements the forces initially exerted by the s-bots result in an immediate translation of the object rather than a rotation. In addition, if an arrangement is stable from the beginning, all s-bots can perceive the target during the whole duration of the transport. On the contrary, if a structure rotates, s-bots may lose visual contact with the target. ${ }^{3}$ Consequently, the performance is likely to decrease.

In the only symmetric case (arrangement $A_{9}$ ), the lowest

\footnotetext{
${ }^{3}$ Note that, in the experiment presented in this section, the omni-directional camera on top of the robot was not yet available.
} 


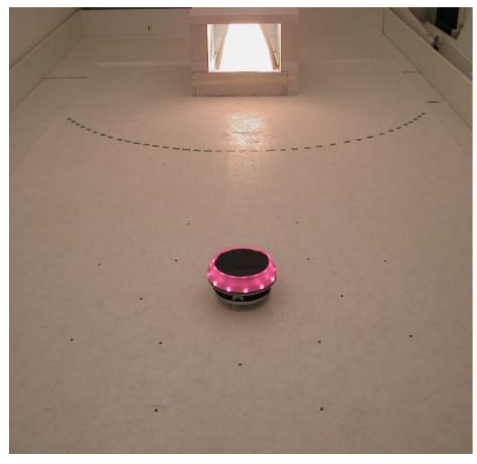

(a)

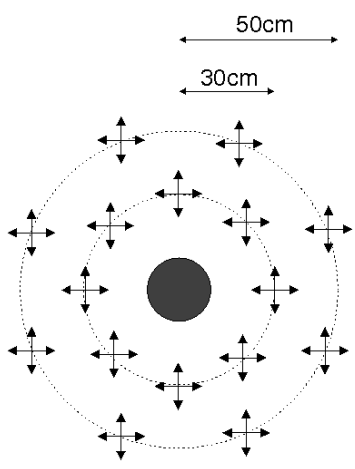

(b)
Fig. 5. Experimental setup: (a) overview of the arena with the object located at a distance of $225 \mathrm{~cm}$ from a light source which represents the center of a circular target zone; (b) potential starting points and orientations of the s-bots around the object.

transport distance observed over all trials on ground $G_{0}\left(G_{1}\right)$ is still $67 \%(54 \%)$ of the distance a single s-bot moving straight without any load can cover within the same amount of time.

\section{Transport by MOdular Robots that SELF-ASSEMBLE}

In this section we consider the case in which the s-bots are initially randomly scattered in the environment. We aim at controlling the modules so that they autonomously form modular robots which in turn manipulate the environment.

\section{A. Experimental Setup}

The manipulation task requires the s-bots to locate, approach, and grasp the object-that has to be subsequently transported from its initial location to a target zone. The experimental setup is shown in Fig. 5a. The object is initially located at a distance of $225 \mathrm{~cm}$ from a light source which represents the center of a circular target zone. The group is considered to be successful if the s-bots manage to move the object inside the target zone within $300 \mathrm{~s}$. If moved in a straight line, the distance covered by the object to enter the target zone is $125 \mathrm{~cm}$.

At the beginning of each trial, six s-bots are positioned in the vicinity of the object. We assume the light source to be strong enough to allow all s-bots to detect the direction to the target zone at every control cycle. The initial position of each s-bot is assigned randomly by uniformly sampling without replacement from a set of 16 specific starting points. The s-bots initial orientation is chosen randomly from a set of four specific directions. The 64 potential placements $(16 \cdot 4)$ of a single s-bot are illustrated in Fig. 5b. The s-bots do not have any knowledge about their starting positions.

The object's weight $(2310 \mathrm{~g})$ is such that a group of four s-bots may not always be sufficient to perform the task. In fact, the performance depends on the way in which the sbots are connected to the object and/or to each other. Four sbots, connected in a "star-like" formation around the object can move it with an average speed of about $1 \mathrm{~cm} \mathrm{~s}^{-1}$. Regardless

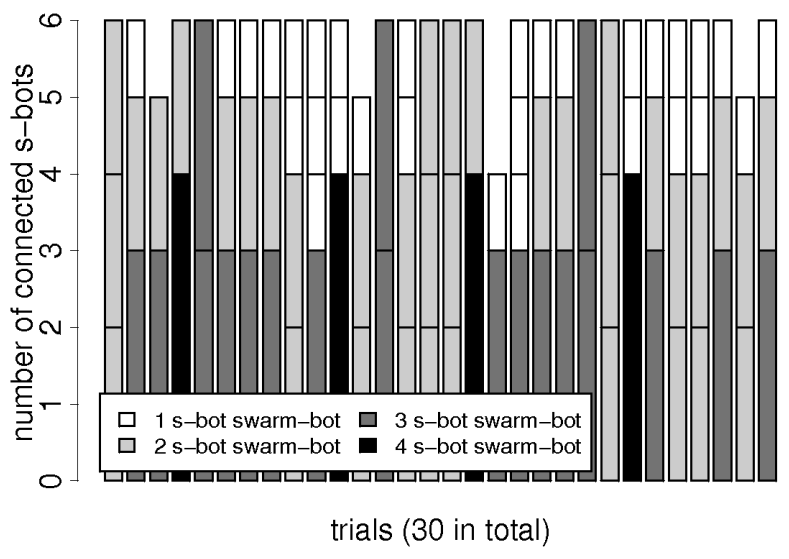

Fig. 7. Number and size of modular robots connected to the object.

the particular arrangement, a group of six s-bots pulling and/or pushing the object is always capable of moving the object.

\section{B. Experimental Results}

In this section, we report data which represents a quantitative description of the performance of the s-bots engaged in the cooperative transport task. Recall that in this task, six s-bots are required to self-assemble and transport the object from its initial position to a target zone. A trial can be divided in two different phases. In the first phase, the s-bots are controlled by the assembly module. Thus, they try to establish a connection either directly to the object or indirectly, via other s-bots. To enable all s-bots to establish a connection before the object starts moving, connected s-bots transport the object only when they do not perceive any unconnected teammates (i.e., if no blue object is perceived; see also Section II-B.1). The assembly phase terminates once every s-bot has successfully established a connection. In the subsequent phase, all s-bots are controlled by the transport module to push/pull the object towards the target. This phase terminates when the object enters the target zone.

We repeated the experiment 30 times. A trial begins with the s-bots randomly placed around the object, and it ends (a) successfully if the s-bots manage to transport the object inside the target zone within the time limit (i.e., $300 \mathrm{~s}$ ), or (b) unsuccessfully if, for any reason, the s-bots fail to transport the object to the target-zone within the time limit. Fig. 6 shows a sequence of three pictures taken from a successful trial.

Fig. 7 illustrates the number and size of the modular robots formed by self-assembly, each of which was connected with the object at the end of each trial. In the first trial, for instance, three modular entities of two s-bots each were engaged in the transport. In trials $4,11,17$, and 24, swarm-bots of four sbots were formed (for an example, see Fig. 6c). Note that the number of swarm-bots, as well as their size and structure, are not explicit. Instead they are emergent properties of the system. Certainly, they depend on the initial spatial arrangement of the modules and on the object's characteristics (e.g., its shape and dimension). However, they are also affected by random components in the s-bots' sensors and actuators. 


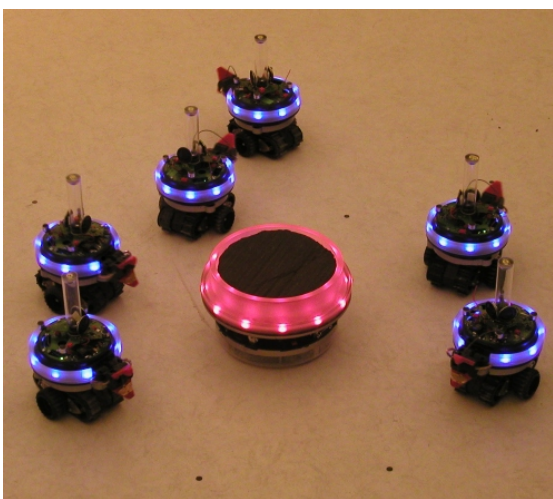

(a)

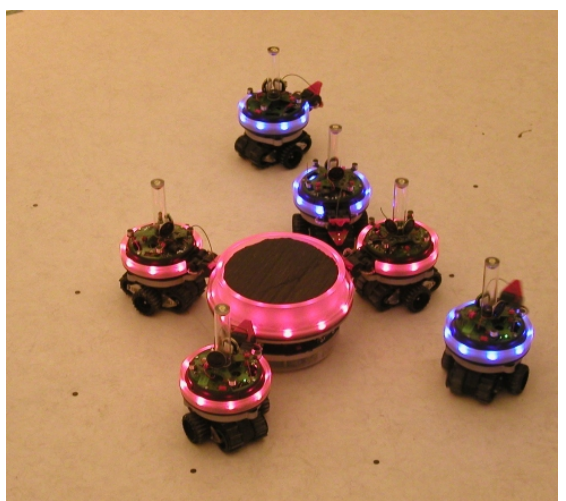

(b)

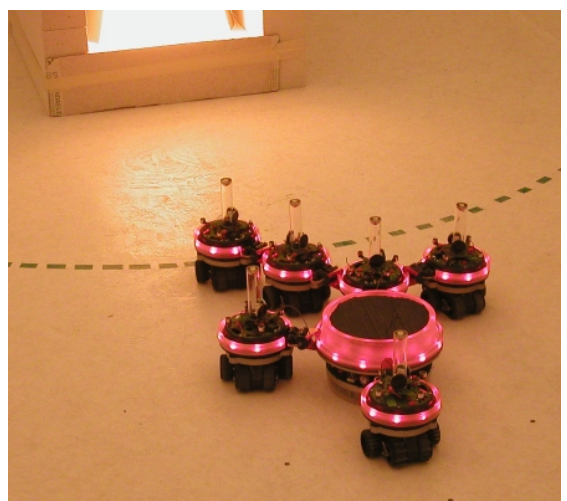

(c)

Fig. 6. These pictures show a sequence of actions, during a trial, in which a group of six s-bots randomly placed around the object (a), initially locates, approaches and connects to the object (b) and finally, once assembled, transports the object to the target zone (c).

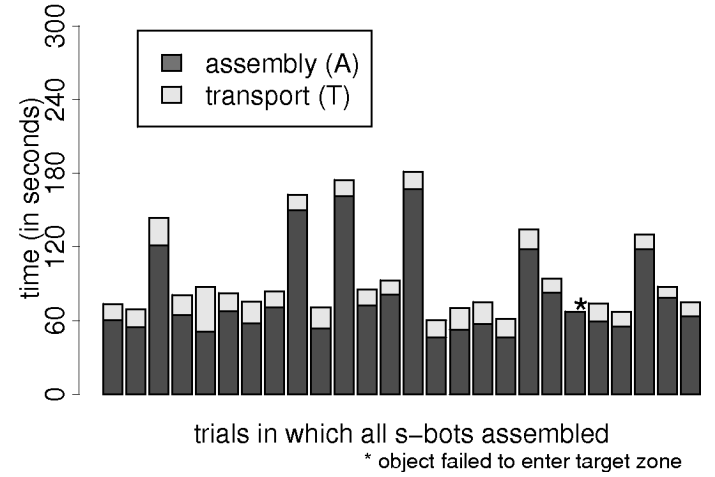

Fig. 8. Time necessary for a group of six s-bots to self-assemble and transport the object inside the target zone.

In 26 out of 30 trials, all six s-bots connected. Out of the 180 connections required by the 30 trials-i.e., six connections per trial times 30 trials-we recorded only 5 failures. Due to one or two s-bots that remain unconnected, in 4 out of 30 trials the s-bots did not manage to reach the transport phase. In fact, in these unsuccessful trials, several s-bots did not activate the transport module as they perceived an unconnected s-bot. Recall that connected s-bots start transporting the object only if they do not perceive any unconnected teammate.

Fig. 8 shows the amount of time per trial spent by the sbots in the two phases of the experiments, that is, assembly and transport. Data concerning the four unsuccessful trials in which one or more s-bots fail to establish a connection are not shown. In 20 out of the 26 trials, the whole group could successfully self-assemble within $83 \mathrm{~s}$, in the other trials selfassembly was successfully completed within $167 \mathrm{~s}$.

Only in a single case out of those in which the s-bots connected successfully, the group failed to transport the object entirely inside the target zone. In this unsuccessful trial, the transport was interrupted in the proximity of the target zone. This failure during the transport phase was probably due to the light reflections in the immediate vicinity of the light source. In fact, a too high intensity of the light disrupts the mechanism used by each s-bot to establish the direction of movement. Therefore, it may happen that, in the immediate vicinity of the target, the entire group loses efficiency in moving the object.

In all other cases, the object entered the target zone within a short period of time; the average transport speed was $8.20 \mathrm{~cm} \mathrm{~s}^{-1}$, which is about $55 \%$ of the maximum speed of a single s-bot moving without any load. Note that the average transport speed is eight times faster than the speed observed for the group of four s-bots, connected in a "star-like" formation.

\section{CONCLUSions}

This paper reported on a series of experiments that present a first attempt to perform a manipulation task by non-stationary self-reconfigurable robots. The manipulation consists in the transport of an object towards a target location.

First, we conducted an experiment in which the modules were manually connected to the object and to each other. Each module was programmed to detect the target, to pull the object, and to align its traction system accordingly. This simple, homogeneous control strategy does neither require the ability to communicate, nor any knowledge about the spatial arrangement of modules. Overall, the strategy proved effective in about 500 trials with 1 to 3 physical modules each. A further analysis revealed that the transport performance is best for grounds on which the traction system has a moderate grip. High friction coefficients $(>1)$, however, may cause a significant decrease in the performance. Furthermore, we gained some first insights, on the impact of the spatial arrangement of modules on the performance of the group.

In a second phase, we carried out a similar experiment, but having the modules started separately, from random positions in the environment. The modules had to assemble with the object and with each other, prior to transportation. The number of modular robots, their size and structure were emergent properties of the system. Apart from a few cases, in which not all modules correctly assembled, the transport speed was more than half the maximum speed of a single module without any load. Note that the object required the cooperative effort of multiple modules to be moved. In fact, the self-assembled 
modular robots comprising six modules in total transported it on average eight times faster than the group of four modules, connected in a "star-like" formation around the object.

So far, group transport by robots has only proven successful for small group sizes and/or for objects providing enough surface for being directly manipulated by each member of the group [31]. On the contrary, self-assembly offers a scalable mechanism for letting a swarm of robots organize into a modular robot of growing size and strength, capable of manipulating objects at different scales.

Future work has to shed light on the capabilities and limitations of the self-assembling structures. Of particular interest will be the study of control strategies that let modular robots adapt their shape, number and/or type of modules in accordance with the changing demands of the environment.

\section{ACKNOWLEDGMENT}

This work was supported by the "ANTS" project, an "Action de Recherche Concertée" funded by the Scientific Research Directorate of the French Community of Belgium, and by the "SWARM-BOTS Project", funded by the Future and Emerging Technologies program of the European Commission, grant IST-2000-31010. The Swiss participants of the project were supported under grant 01.0012 by the Swiss Government. The information provided is the sole responsibility of the authors and does not reflect the Community's opinion. The Community is not responsible for any use that might be made of data appearing in this publication. Roderich Groß acknowledges additional support from the Japan Society for the Promotion of Science (JSPS). Marco Dorigo acknowledges support from the Belgian FNRS, of which he is a Research Director.

\section{REFERENCES}

[1] M. Yim, Y. Zhang, and D. Duff, "Modular robots," IEEE Spectr., vol. 39, no. 2, pp. 30-34, 2002.

[2] D. Rus, Z. Butler, K. Kotay, and M. Vona, "Self-reconfiguring robots," Commun. ACM, vol. 45, no. 3, pp. 39-45, 2002.

[3] D. Tesar and M. Butler, "A generalized modular architecture for robot structures," Manuf. Rev., vol. 2, no. 2, pp. 91-118, 1989.

[4] C. J. J. Paredis, H. B. Brown, and P. K. Khosla, "A rapidly deployable manipulator system," Robot. Auton. Syst., vol. 21, no. 3, pp. 289-304, 1997.

[5] A. Castano, W.-M. Shen, and P. M. Will, "CONRO: Towards deployable robots with inter-robots metamorphic capabilities," Auton. Robots, vol. 8, no. 3, pp. 309-324, 2000

[6] S. Murata, E. Yoshida, A. Kamimura, H. Kurokawa, K. Tomita, and S. Kokaji, "M-TRAN: Self-reconfigurable modular robotic system," IEEE/ASME Trans. Mechatron., vol. 7, no. 4, pp. 431-441, 2002.

[7] M. Yim, K. Roufas, D. Duff, Y. Zhang, C. Eldershaw, and S. B. Homans, "Modular reconfigurable robots in space applications," Auton. Robots, vol. 14, no. 2-3, pp. 225-237, 2003.

[8] M. W. Jørgensen, E. H. Østergaard, and H. H. Lund, "Modular ATRON: Modules for a self-reconfigurable robot," in Proc. of the 2004 IEEE/RSJ Int. Conf. on Intelligent Robots and Systems, vol. 2. IEEE Computer Society Press, Los Alamitos, CA, 2004, pp. 2068-2073.

[9] K. Tomita, S. Murata, H. Kurokawa, E. Yoshida, and S. Kokaji, "Selfassembly and self-repair method for a distributed mechanical system," IEEE Trans. Robot. Automat., vol. 15, no. 6, pp. 1035-1045, 1999.

[10] S. Murata, E. Yoshida, H. Kurokawa, K. Tomita, and S. Kokaji, "Selfrepairing mechanical systems," Auton. Robots, vol. 10, no. 1, pp. 7-21, 2001.
[11] T. Fukuda and S. Nakagawa, "Method of autonomous approach, docking and detaching between cells for dynamically reconfigurable robotic system CEBOT," JSME Int. J. III-VIB. C., vol. 33, no. 2, pp. 263-268, 1990.

[12] M. Yim, Y. Zhang, K. Roufas, D. Duff, and C. Eldershaw, "Connecting and disconnecting for chain self-reconfiguration with PolyBot," IEEE/ASME Trans. Mechatron., vol. 7, no. 4, pp. 442-451, 2002.

[13] M. Rubenstein, K. Payne, P. Will, and W.-M. Shen, "Docking among independent and autonomous CONRO self-reconfigurable robots," in Proc. of the 2004 IEEE Int. Conf. on Robotics and Automation, vol. 3. IEEE Computer Society Press, Los Alamitos, CA, 2004, pp. 2877-2882.

[14] R. Groß, M. Bonani, F. Mondada, and M. Dorigo, "Autonomous selfassembly in a swarm-bot," in Proc. of the 3rd Int. Symp. on Autonomous Minirobots for Research and Edutainment (AMiRE 2005). Springer Verlag, Berlin, Germany, 2006, pp. 314-322.

[15] M. Yim, D. G. Duff, and K. D. Roufas, "PolyBot: a modular reconfigurable robot," in Proc. of the 2000 IEEE Int. Conf. on Robotics and Automation, vol. 1. IEEE Computer Society Press, Los Alamitos, CA, 2000, pp. 514-520.

[16] A. Castano, A. Behar, and P. M. Will, "The CONRO modules for reconfigurable robots," IEEE/ASME Trans. Mechatron., vol. 7, no. 4, pp. 403-409, 2002.

[17] S. Hirose, T. Shirasu, and E. F. Fukushima, "Proposal for cooperative robot "Gunryu" composed of autonomous segments," Robot. Auton. Syst., vol. 17, pp. 107-118, 1996.

[18] H. B. Brown, M. Weghe, C. Bererton, and P. K. Khosla, "Millibot trains for enhanced mobility," IEEE/ASME Trans. Mechatron., vol. 7, no. 4, pp. 452-461, 2002.

[19] M. Yim, D. G. Duff, and K. D. Roufas, "Walk on the wild side," IEEE Robot. Automat. Mag., vol. 9, no. 4, pp. 49-53, 2002.

[20] K. Støy, W.-M. Shen, and P. Will, "On the use of sensors in selfreconfigurable robots," in Proc. of the $7^{\text {th }}$ Int. Conf. on Simulation of Adaptive Behavior. MIT Press, Cambridge, MA, 2002, pp. 48-57.

[21] A. Kamimura, H. Kurokawa, E. Yoshida, S. Murata, K. Tomita, and S. Kokaji, "Automatic locomotion design and experiments for a modular robotic systems," IEEE/ASME Trans. Mechatron., vol. 10, no. 3, pp. 314-325, 2005.

[22] R. O'Grady, R. Groß, F. Mondada, M. Bonani, and M. Dorigo, "Selfassembly on demand in a group of physical autonomous mobile robots navigating rough terrain," in Proc. of $8^{\text {th }}$ European Conf. on Artificial Life, ser. Lecture Notes in Artificial Intelligence, vol. 3630. Springer Verlag, Berlin, Germany, 2005, pp. 272-281.

[23] T. Fukuda and T. Ueyama, Cellular Robotics and Micro Robotic Systems. World Scientific Publishing, London, UK, 1994.

[24] K. Kotay and D. Rus, "The inchworm robot: A multi-functional system," Auton. Robots, vol. 8, no. 1, pp. 53-69, 2000.

[25] M. Dorigo, "SWARM-BOT: An experiment in swarm robotics," in Proc. of the 2005 IEEE Swarm Intelligence Symposium. IEEE Computer Society Press, Los Alamitos, CA, 2005, pp. 192-200.

[26] F. Mondada, L. M. Gambardella, D. Floreano, S. Nolfi, J.-L. Deneubourg, and M. Dorigo, "The cooperation of swarm-bots: Physical interactions in collective robotics," IEEE Robot. Automat. Mag., vol. 12, no. 2, pp. 21-28, 2005.

[27] R. Groß and M. Dorigo, "Group transport of an object to a target that only some group members may sense," in Proc. of the $8^{\text {th }}$ Int. Conf. on Parallel Problem Solving from Nature, ser. Lecture Notes in Computer Science, vol. 3242. Springer Verlag, Berlin, Germany, 2004, pp. 852861.

[28] R. Groß, F. Mondada, and M. Dorigo, "Transport of an object by six pre-attached robots interacting via physical links," in Proc. of the 2006 IEEE Int. Conf. on Robotics and Automation. IEEE Computer Society Press, Los Alamitos, CA, 2006, this proceedings.

[29] R. Groß, M. Bonani, F. Mondada, and M. Dorigo, "Autonomous selfassembly in mobile robotics," IRIDIA - Université Libre de Bruxelles, Tech. Rep. IRIDIA/2005-2, 2005.

[30] J. M. Chambers, W. S. Cleveland, B. Kleiner, and P. A. Tukey, Graphical Methods for Data Analysis, ser. The Wadsworth statistics/probability series. Wadsworth \& Brooks/Cole, Pacific Grove, CA, 1983.

[31] C. R. Kube and E. Bonabeau, "Cooperative transport by ants and robots," Robot. Auton. Syst., vol. 30, no. 1-2, pp. 85-101, 2000. 